Байкальский государственный университет, г. Иркутск, Российккая Федерация

Н. Н. Грибанова

Байкальский государственный университет, г. Иркутск, Российкая Федерация

\title{
ТЕОРЕТИЧЕСКИЕ ПОДХОДЫ \\ К ПЛАНИРОВАНИЮ И ПРОГНОЗИРОВАНИЮ ЭКОНОМИЧЕСКОЙ ДЕЯТЕЛЬНОСТИ КОМПАНИИ
}

\begin{abstract}
АНнОтАЦИЯ. Прогнозирование деятельности хозяйствующего субъекта связано с тем, что мониторинг движения материальных и денежных потоков позволяет определить финансовые возможности предприятия в краткосрочной, а также в долгосрочной перспективе. Выяснить, достаточно ли собственных средств для покрытия всех текущих расходов и защиты компании от влияния негативных внешних факторов, а также для обеспечения финансовой стабильности и достижения высокого результата финансово-хозяйственной деятельности. В современных условиях важной областью стало информационное обеспечение в хозяйственной деятельности компании, которое состоит в сборе и переработке информации, необходимой для принятия обоснованных управленческих решений. Полученная информация служит основой для подготовки соответствующих докладов, отчетов, предложений для выработки и принятия стратегических и тактических решений по управлению компанией.
\end{abstract}

кЛючЕВЫЕ словА. Планирование; прогнозирование; бюджетирование; моделирование; стратегия.

ИНФОРМАЦИЯ О СТАТЬЕ. Дата поступления 18 апреля 2016 г.; дата принятия к печати 13 мая 2016 г.; дата онлайн-размещения 26 июля 2016 г.

\author{
N. S. Gribanova \\ Baikal State University, \\ Irkutsk, Russian Federation \\ N. N. Gribanova \\ Baikal State University, \\ Irkutsk, Russian Federation
}

\section{THEORETICAL APPROACHES TO PLANNING AND FORECASTING OF COMPANY'S ECONOMIC ACTIVITY}

\begin{abstract}
Forecasting of an economic entity's activity is connected with the fact that monitoring of material and cash flows allows to identify financial possibilities of an enterprise both in short-term and long-term prospects, to find out whether the equity funds are sufficient for covering all current expenditures and protecting the company from the impact of negative external factors, as well as for providing financial stability and achieving high performance in financial and economic activity. In the current context, of an important area has become information support in the company's economic activity which involves collection and processing the information needed for making substantiated managerial decisions. The information gained serves as a basis for preparing relevant reports, accounts, proposals for working out and making strategic and tactical decisions on company management.

KEYWORDS. Planning; forecasting; budgeting; modeling; strategy.
\end{abstract}

ARTICLE INFO. Received April 18, 2016; accepted May 13, 2016; available online July 26, 2016.

(C) Н. С. Грибанова, Н. Н. Грибанова, 2016

\section{Baikal Research Journal}


Планирование как наука представляет собой комплекс систематизированных знаний о закономерностях развития и функционирования различных хозяйственных систем. Наука планирования - многоотраслевая. Отдельные ее разделы, например, оперативно-календарное планирование, всесторонне разработаны. Менее разработано тактическое и стратегическое планирование в компании. Это говорит о том, что наука планирования в целом пока находится в стадии формирования [1, с. 201].

Стратегическое планирование обеспечивает основу для всех управленческих решений, а функции организации, мотивации и контроля ориентированы на выработку стратегических планов.

В стратегическом планировании важным аспектом выступает анализ перспектив организации, выделяются опасности, возможности, тенденции, которые могут повлиять на уже сложившиеся и существующие в определенный период времени тенденции. Стратегическое планирование является инструментом, при помощи которого создается система целей функционирования предприятия и объединяются усилия всего коллектива по ее достижению. Его важнейшая задача - обеспечить нововведения, необходимые для деятельности предприятия, а также улучшить адаптацию организации к внешней среде [2, с. 185-187].

Экономисты, не связанные с теорией автоматизации информационных потоков, определением «кирпичик» информации (операции), как правило, подходят к рассмотрению вопроса прогнозирования хозяйственной деятельности хозяйствующего субъекта с позиций наличия собственных оборотных средств, степени соответствия фактических запасов активов нормативным, величине затрат и прибыли, т. е. рассматривают финансовые отношения в компании без учета технологического процесса.

Наиболее важный этап анализа финансового состояния компании заключается в определении наличия собственных и приравненных к ним средств, а также в установлении факторов, влияющих на их изменение в определенном периоде. Положительным считается превышение дебиторской задолженности над кредиторской, а наиболее оптимальным вариантом - формула отсутствия задолженности как самой компании, так и других хозяйствующих субъектов.

Теоретически вопросы построения финансовых прогнозов в экономической науке подразделяются на деление прогнозных оценок в зависимости от продолжительности периода, к которому относится разрабатываемый прогноз. Это следующие периоды:

- стратегический;

- долгосрочный;

- среднесрочный;

- годовой;

- оперативный.

Подобное деление разработано экономистами-теоретиками без учета современных электронно-математических методов при исследовании операций в экономике и носит механистический, негибкий подход к исследуемой проблеме хозяйствования.

В работах авторов с подобным подходом присутствует методика расчетов укрупненных показателей на основе статей баланса, что не может в полной мере определить источники экономии, которые можно найти на основе теории исследования операций [3, с. 67-69].

Финансовый прогноз экономисты-теоретики предлагают строить на основе трех основных типах моделей:

- дескриптивные;

- предикативные;

- нормативные.

Дескриптивные модели - основные модели описательного характера, используемые для анализа финансового состояния компании. $К$ ним относятся разработка

\section{Baikal Research Journal}


системы отчетных балансов, представление финансовой отчетности в различных аналитических разрезах, вертикальный, горизонтальный анализ отчетности, система аналитических коэффициентов, аналитические записки к отчетности.

Предикативные модели - это модели предсказательного, прогностического характера. Наиболее распространенными из них являются расчет точки критического объема продаж, построение прогностических финансовых отчетов, модели динамического анализа (жестко детерминированные факторные модели и регрессионные модели), модели ситуационного анализа.

С помощью нормативных моделей предоставляется возможность сравнивать фактические результаты деятельности компании с ожидаемыми, рассчитанными по бюджету. В основном данные модели используются во внутреннем финансовом анализе. Суть их состоит в определении нормативов по каждой статье расходов по технологическим процессам, видам изделий, центрам ответственности и анализа отклонений фактических данных от этих нормативов [4, с. 165].

- в экономическом анализе деятельности компании предлагаются следующие основные подходы:

- вертикальный анализ - представляет финансовый отчет в виде относительных показателей;

- горизонтальный анализ - позволяет построить одну или несколько аналитических таблиц, в которых абсолютные балансовые показатели дополняются относительными темпами роста;

- трендовый анализ - часть перспективного анализа, необходим в управлении для финансового прогнозирования. Это самый простой способ финансового прогнозирования;

- факторный анализ - предполагает, что результативный показатель зависит от разнообразных и многочисленных факторов. Под факторным анализом понимается методика комплексного и системного изучения и измерения воздействия факторов на величину результативных показателей;

- анализ с помощью финансовых коэффициентов [5, с. 215].

В литературе по планированию в компании также различают две схемы организации работ по составлению бюджетов: по методу break-down (сверху-вниз) и по методу build-up (снизу-вверх). По первому методу break-down работа по составлению бюджетов начинается «сверху», т. е. руководство компании устанавливает цели и задачи, в частности плановые показатели по прибыли [6, с. 135]. После чего данные показатели во все более детализированной форме (по мере продвижения на более низкие уровни структуры предприятия) включаются в планы подразделений.

По методу build-up расчет показателей реализации начинаются с отдельных сбытовых подразделений, а затем уже руководитель отдела реализации предприятия сводит данные показатели в единый бюджет, который впоследствии может войти составной частью в общий план предприятия [7, с. 75].

Наиболее важной характеристикой процесса прогнозирования является его неопределенность. На практике точность прогноза определяется в конечном итоге сочетанием двух компонентов:

- во-первых, это адекватность используемой модели, т. е. насколько точно она отражает реальное состояние исследуемого объекта;

- во-вторых, это наличие достаточного объема объективной исходной информации для построения прогноза.

В основном при прогнозировании объемов производства показатели определяются с помощью экстраполяции тенденций развития процессов и явлений из прошлого в будущее. Глубина используемой информации при построении тренда зависит от начала развития тенденций и от переломного периода в общей тенденции.

\section{Baikal Research Journal}


В прогнозе также используются кроме экстраполируемых данных удельные показатели и нормативы.

Однако при подобных подходах в прогнозировании деятельности компании остается высокой неопределенность прогнозных оценок. Под неопределенностью прогноза понимается величина максимально возможной ошибки или погрешности, т. е. отклонения фактического состояния объекта от того, которое предсказывалось в прогнозе.

Проанализируем последовательность и содержание этапов одного цикла экономико-математического моделирования.

Постановка экономической проблемы и ее качественный анализ. На первом этапе важно точно сформулировать суть проблемы и принимаемые допущения. Данный этап включает определение наиболее важных черт и свойств моделируемого объекта и абстрагирование от второстепенных задач. На этом этапе изучается структура объекта и основные экономические зависимости, объясняющие поведение и развитие объекта [8, с. 215].

Подходы при анализе деятельности компании показывают отсутствие точных критериев для постановки экономической задачи для моделирования процессов. Следовательно, переход к следующему этапу, а именно к построению математической модели, невозможен. Однако целая армия математиков от экономики предлагает модели исследования экономических процессов на основе недостоверной информации, пытаясь ее хоть как-то превратить в базу для анализа прогнозных оценок.

На этом этапе предлагается математический анализ модели. Целью этапа является выделение общих свойств модели. В данном случае применяются только математические приемы исследования. Очень часто анализ математической модели ведется от противного. Если получится доказать, что математическая задача не имеет ни одного решения, то надобность в дальнейшей работе по первоначальному варианту модели отпадает и, из этого следует, что необходимо скорректировать постановку экономической задачи или способы ее математической формализации [9, с. 97].

При аналитическом исследовании модели раскрываются такие вопросы, как, например, единственно ли решение, какие переменные (неизвестные) могут входить в решение, какие будут соотношения между ними, в каких пределах и в зависимости от каких исходных условий они изменяются, каковы тенденции их изменения и т. д. В данном случае видна разница в подходах к решению проблемы: экономический подход - это чисто качественный, математический - чисто количественный.

Использование общих свойств моделей имеет важное значение и исследуется посредством идеализации первоначальных моделей этих свойств. Но, тем не менее, сложные экономические модели трудно подвергаются аналитическому анализу.

Экономико-математическая модель способствует математическому описанию экономического процесса, а именно, описанию факторов, составляющих структуру и закономерности изменения данного экономического явления с помощью математических символов и приемов (уравнений, неравенств, таблиц, графиков и т. д.). Построение модели возможно и по функциональной, и по корреляционной связям. Функциональная связь выражается уравнением вида:

$$
Y=f(x),
$$

где $y-$ показатель; $x-$ факторы.

Корреляционные модели позволяют определить связь размера продаж от изменений внешней среды предприятия, в том числе макроэкономических переменных (темпы роста валового внутреннего продукта, темпа инфляции и т. д.), а также от отраслевых показателей (состояния отрасли, уровня конкуренции в ней, емкости отраслевого рынка) [10, с. 125].

\section{Baikal Research Journal}


Еще один метод, применяемый в системе прогнозных оценок - это так называемый эвристический метод, который основывается на усреднении сведений, получаемых при интервьюировании разных участков рынка: работников торговли, специалистов маркетинговых служб, покупателей. Главной чертой данного метода является то, что он представляет достоверную информацию о нуждах потребителя, но не учитывает вероятность изменения рыночной конъюнктуры [11, с. 123].

Для учета временных изменений объема продаж продукции, работ и услуг применятся метод анализа временных рядов, который включает в себя метод экстраполяции, анализ сезонности, анализ цикличности.

Суть метода экстраполяции состоит в распространении выводов, полученных на основе наблюдений за объемом продаж на будущее в течение определенного периода времени $[12$, с. 283]. По нашему мнению, добиться устойчивого состояния предприятия на рынке можно только на основании построения алгоритма расчета, основываясь на простейшем «кирпичике» деятельности хозяйствующего субъекта - операции и ее оценки как во временном, так и в финансовом планах. Данный подход основывается на построении прогнозов «снизу» с максимальной детализацией производственного процесса. Быстродействие персональных компьютеров на сегодняшний день уже не является ограничением для построения подобных расчетов.

Основной задачей предложенного подхода будут границы применимости моделей прогнозирования и вероятность предлагаемой прогнозной оценки. Следствием работы предложенной программы явится экономия времени при принятии управленческих решений, также разрабатывается и система возможных погрешностей в процессе прогнозирования управленческих задач.

Для построения автоматизированной системы прогнозирования деятельности предприятия на первый план выступает нормирование труда, запасов, электроэнергии и теплоэнергии, производственных и непроизводственных расходов, то есть, всей системы деятельности компании. Поэтому при внедрении подобных систем автоматизации прогнозирования часто приходится отказываться от существующей системы управления, что влечет за собой изменение движения информации, менталитета работников предприятия на всех структурных уровнях управления.

На сегодняшний день во многих компаниях среднего звена уже существующая система внутрифирменного прогнозирования не подразумевает многовариантный анализ финансовых последствий реализации намеченных планов, не предполагает анализ различных сценариев изменения финансового состояния фирмы. Учитывая это, можно подчеркнуть, что жестко предопределенных и однозначных решений в этих компаниях нет.

Следует отметить, что роль и предназначение прогнозирования как функции организации деятельности любой экономической системы не в точном расчете тех или иных показателей, а в определении направления стратегии предприятия в быстро меняющихся внешних условиях хозяйствования. Эта работа призвана обнаружить внутренние резервы компании, соблюдать режим экономии. Получение прогнозного размера прибыли и других финансовых показателей возможно лишь при условии соблюдения норм затрат труда и материальных ресурсов. Следствием этих расчетов является устранение чрезмерных запасов материальных ресурсов, сокращение непроизводительных расходов, внеплановых финансовых инвестиций. Благодаря расчету прогноза деятельности предприятия создаются необходимые условия для эффективного использования производственных мощностей, повышения качества продукции.

Для осуществления автоматизации прогнозных оценок на предприятии необходимо иметь или разработать комплект технологической и конструкторской документации, которая касается организации технологического процесса производства.

Технологические процессы создаются технологом в виде плана-графика, где устанавливаются этапы освоения изделия, перечень работ по технологической под-

\section{Baikal Research Journal}

электронный научный журнал Байкальского государственного университета 
готовке производства и длительность их выполнения, состав подразделений-исполнителей и ответственные по каждому подразделению. Без этих данных разработка автоматизированного процесса прогнозирования значительно потеряет точность в расчетах.

Основными методами расчетов трудозатрат и, в конечном счете, стоимости и других характеристик изделий выступает функционально-стоимостной анализ (ФСA, ActivityBasedCosting, АВС), использующий в качестве основы функции и ресурсы, задействованные в производстве, продаже, оказании услуг, технической поддержке, маркетинге, доставке, обеспечении качества [13, с. 115].

В заключение отметим, что на сегодняшний день автоматизация процессов охватила многие отрасли промышленности и сферы деятельности: от производственных процессов до совершения покупок в магазинах. Вне зависимости от размера и сферы деятельности организации практически в каждой компании существуют автоматизированные процессы.

Основной целью автоматизации любой деятельности человека является повышение качества исполнения процесса. Автоматизированный процесс обладает более стабильными характеристиками, чем процесс, осуществляемый в ручном режиме. Несмотря на то, что автоматизация процессов может выполняться на различных уровнях, принципы автоматизации для всех уровней и всех видов процессов будут оставаться едиными. Это общие принципы, которые задают условия эффективного выполнения процессов в автоматическом режиме и устанавливают правила автоматического управления процессами.

\section{Список использованной литературы}

1. Бухалков М. И. Планирование на предприятии : учеб. пособие / М. И. Бухалков. М. : Инфра-М, 2011. - 410 с.

2. Кузык Б. Н. Прогнозирование, стратегическое планирование и национальное программирование : учеб. пособие / Б. Н. Кузык, В. И. Кушлин, Ю. В. Яковец. - М. : Экономика, 2011. - $604 \mathrm{c}$.

3. Акчурина Е. В. Бухгалтерский финансовый учет : учеб. пособие / Е. В. Акчурина, Л. П. Солодка. - М. : Экзамен, 2013. - 178 с.

4. Морошкин В. А. Бизнес-планирование : учеб. пособие / В. А. Морошкин, В. П. Буров. - М. : Форум, 2011. - 287 с.

5. Вайс Е. С. Планирование на предприятии организации : учеб. пособие / Е. С. Вайс, В. М. Васильцова. - М. : КноРус, 2012. -336 с.

6. Грибанова Н. Н. Планирование и бюджетирование деятельности малого предприятия : учеб. пособие / Н. Н. Грибанова, Ю. И. Колесник. - Иркутск : Изд-во БГУЭП, 2014. - 210 с.

7. Басовский Л. Е. Прогнозирование и планирование в условиях рынка : учеб. пособие / Л. Е. Басовский. - М. : Инфра-М, 2013. - 156 с.

8. Беляев В. К. Планирование на предприятии : учеб. пособие / В. К. Беляев. - Иркутск : Изд-во БГУЭП, 2011. - 100 с.

9. Вологнина О. А. Математическое моделирование экономических процессов и систем : учеб. пособие / О. А. Волгнина. - М. : КноРус, 2012. - 196 с.

10. Савиных В. Н. Математическое моделирование производственного и финансового менеджмента : учеб. пособие / В. Н. Савиных. - М. : КноРус, 2014. — 192 с.

11. Стрекалова Н. Д. Бизнес-планирование: теория и практика : учеб. пособие / Н. Д. Стрекалова. - СПб. : Питер, 2013. - 352 с.

12. Кокаева Т. Т. О некоторых аспектах использования информационных технологий в управлении малыми предприятиями / Т. Т. Кокаева, А. Е. Бердникова, Т. Т. Бугулова // Известия Горского государственного аграрного университета. - 2013. - Т. 50, ч. 1 (85). C. 285-288.

13. Самаруха А. В. Прогнозирование регионального развития при переходе России на инновационную модель экономики : учеб. пособие / А. В. Самаруха. - Иркутск : Изд-во БГУЭП, 2013. - 240 c.

\section{Baikal Research Journal}




\section{References}

1. Bukhalkov M. I. Planirovanie na predpriyatii [Corporate planning]. Moscow, Infra-M Publ., 2011. 410 p.

2. Kuzyk B. N., Kushlin V. I., Yakovets Yu. V. Prognozirovanie, strategicheskoe planirovanie i natsional'noe programmirovanie [Forecasting, strategic planning and national programming]. Moscow, Ekonomika Publ., 2011. 604 p.

3. Akchurina E. V., Solodka L. P. Bukhgalterskii finansovyi uchet [Business financial accounting]. Moscow, Ekzamen Publ., 2013. 178 p.

4. Moroshkin V. A., Burov V. P. Biznes-planirovanie [Business planning]. Moscow, Forum Publ., 2011. $287 \mathrm{p}$.

5. Vais E. S., Vasil'tsova M. Planirovanie na predpriyatii organizatsii [Planning in an organization's enterprise, Moscow, KnoRus Publ., 2012. 336 p.

6. Gribanova N. N., Kolesnik Yu. I. Planirovanie i byudzhetirovanie deyatel'nosti malogo predpriyatiya [Planning and budgeting of the small business's activity]. Irkutsk, Baikal State University of Economics and Law Publ., 2014. 210 p.

7. Basovsky L. E. Prognozirovanie i planirovanie $v$ usloviyakh rynka [Forecasting and planning in market environment]. Moscow, Infra-M Publ., 2013. $156 \mathrm{p}$.

8. Belyayev V. K. Planirovanie na predpriyatii [Corporate planning]. Irkutsk, Baikal State University of Economics and Law Publ., 2011. 100 p.

9. Volognina O. A. Matematicheskoe modelirovanie ekonomicheskikh protsessov i sistem [Mathematical modelling of economic processes and systems]. Moscow, KnoRus Publ., 2012. 196 p.

10. Savinykh V. N. Matematicheskoe modelirovanie proizvodstvennogo $i$ finansovogo menedzhmenta [Mathematical modelling of industrial and financial management]. Moscow, KnoRus Publ., 2014. 192 p.

11. Strekalova N. D. Biznes-planirovanie: teoriya i praktika [Business planning: theory and practice]. Saint Petersburg, Piter Publ., 2013. 352 p.

12. Kokayeva T. T., Bugulova T. T. On some aspects of using information technologies in management of small businesses. Izvestiya Gorskogo gosudarstvennogo agrarnogo universiteta $=$ Proceedings of Gorsky State Agrarian University, 2013, vol. 50, pr. 1 (85), pp. 285-288. (In Russian).

13. Samarukha A. V. Prognozirovanie regional'nogo razvitiya pri perekhode Rossii na innovat sionnuyu model' ekonomiki [Forecasting of regional development in Russia's transition to innovative model of economy]. Irkutsk, Baikal State University of Economics and Law Publ., 2013. 240 p.

\section{Информация об авторах}

Грибанова Наталия Сергеевна - аспирант, кафедра экономики предприятия и предпринимательской деятельности, Байкальский государственный университет, 664003, г. Иркутск, ул. Ленина, 11, e-mail: gribanova1992@list.ru.

Грибанова Нина Николаевна - кандидат экономических наук, доцент, кафедра экономики предприятия и предпринимательской деятельности, Байкальский государственный университет, 664003, г. Иркутск, ул. Ленина, 11, e-mail: miss.gribanova@bk.ru.

\section{Authors}

Natalia S. Gribanova - PhD student, Chair of Enterprise and Entrepreneurship Economy, Baikal State University, 11 Lenin St., 664003, Irkutsk, Russian Federation; e-mail: gribanova1992@list.ru.

Nina N.Gribanova - PhD in Economics, Associate Professor, Chair of Enterprise and Entrepreneurship Economy, Baikal State University, 11 Lenin St., 664003, Irkutsk, Russian Federation; e-mail: miss.gribanova@bk.ru.

\section{Библиографическое описание статьи}

Грибанова Н. С. Теоретические подходы к планированию и прогнозированию экономической деятельности компании / Н. С. Грибанова, Н. Н. Грибанова // Baikal Research Journal. — 2016. — T. 7, № 4. — DOI : 10.17150/2411-6262.2016.7(4).16.

\section{Reference to article}

Gribanova N. S., Gribanova N. N. Theoretical approaches to planning and forecasting of company's economic activity. Baikal Research Journal, 2016, vol. 7, no. 4. DOI : 10.17150/24116262.2016.7(4).16. (In Russian).

\section{Baikal Research Journal}

